\title{
SYMMETRY BREAKING PHENOMENA OF PURELY VISCOUS SHEAR-THINNING FLUID FLOW IN A LOCALLY CONSTRICTED CHANNEL
}

\author{
Ternik, P. \\ University of Maribor, Faculty of Mechanical Engineering, \\ Smetanova ulica 17, 2000 Maribor, Slovenia \\ E-Mail: primoz.ternik@uni-mb.si
}

\begin{abstract}
The goal of a present study is to investigate the effects of generalized Newtonian fluids on the threshold of the transition from flow symmetry to its asymmetry for the flow through a locally constricted channel. We consider purely viscous shear-thinning fluid and compare it with the Newtonian fluid. Fluid flow is studied numerically by solving the two dimensional momentum equations along with the continuity equation and the Carreau-Yasuda rheological model. We report systematic results in a range of generalized Reynolds number $150 \leq \operatorname{Re} \leq 250$ with a focus on its critical value. Results indicate that the shear-thinning viscous behaviour decreases the onset of bifurcation phenomena and the critical value of Reynolds number. Last but not least, a systematic grid refinement analysis and numerical accuracy study is performed and present numerical results may be treated as the benchmark. (Received in January 2008, accepted in August 2008. This paper was with the author 3 months for 1 revision.)
\end{abstract}

Key Words: Bifurcation, Inelastic Shear-Thinning Fluid, Carreau-Yasuda Model, Numerical Modelling

\section{INTRODUCTION}

In recent years it has been of great interest to study the fluid flow in channels experiencing the transition from symmetry to asymmetry. Examples of such a flow, among others, are found in heat exchangers, ducts for industrial use and flows around buildings. To aid in experimental and numerical investigations, the flow in a sudden symmetric expansion has been recognized as a representative test bed because it involves a configuration which is regarded as having one of the simplest geometries. But on the other hand, geometrical simplicity does not imply that the flow phenomena are also simple. Moreover, flow separation and reattachment as well as multiple recirculating regions of fluid flow are those rich features that attracted the interest of many researchers.

For Newtonian flows through suddenly expanded channels it has been determined (both experimentally [1, 2] and numerically [2]) that the initially symmetric flow becomes asymmetric as the Reynolds number is increased beyond a critical value $\left(R e_{c r}\right)$, with recirculation zones of different sizes. With further increase of the Reynolds number, the flow becomes time dependent and three-dimensional, and finally turbulent.

With respect to inelastic non-Newtonian fluid flows in a two-dimensional geometry with symmetric geometrical changes (expansions), it is reported that the non-Newtonian rheology greatly influences not only the vortex size and reattachment length [3] but also the onset of bifurcation (transition from initial symmetry to asymmetry) as well as the vortices size in a point of bifurcation; shear-thickening viscous behaviour decreases the critical value of Reynolds number [4], while the shear-thinning viscous behaviour delays the onset of the 
bifurcation (increases the critical value of Reynolds number) and pronounces flow asymmetry [5] in comparison to Newtonian fluids.

In spite of an exhaustive studies (experimental and numerical) of generalized Newtonian fluid flows through sudden symmetric expansions of various expansion ratios almost no computational results regarding the generalized Newtonian fluid flow through a channel with local constriction has been reported so far. The present work therefore aims at the numerical study of Newtonian and shear-thinning fluid flow in such a geometry with a focus on bifurcation phenomena and influence of shear-thinning viscous behaviour on this phenomena. Thorough and detailed numerical study constitutes a comprehensive set of results to which other numerical simulations can be rigorously compared.

As a first step, the numerical analysis of fully developed Carreau-Yasuda fluid flow in a channel is performed in order to estimate the generalized Reynolds number. The present work is followed with a thorough and systematic numerical modelling of generalized Newtonian fluid flow in a locally constricted channel.

The remaining sections of this paper are organized as follows: working equations and problem specifications are given in Section 2 and 3. This is followed by a brief description of a numerical procedure. The numerical results, together with the nodalization analysis and conclusions are presented in the remaining sections.

\section{MATHEMATICAL MODEL}

In a present study the fluid flow was assumed to be steady, laminar, incompressible and generalized Newtonian. The mathematical model for such a flow in the locally constricted channel with rigid walls uses the Navier-Stokes equations which are generalized to take into account the shear-dependent rheological behaviour of a fluid.

\subsection{Governing equations}

Mass and momentum balance laws for the incompressible viscous fluid flow in the Cartesian co-ordinates frame of reference can be written in the convection-diffusion form as:

$$
\begin{gathered}
\frac{\partial v_{i}}{\partial x_{i}}=0 \\
\rho v_{j} \frac{\partial v_{i}}{\partial x_{j}}-\frac{\partial}{\partial x_{j}}\left[\eta(\dot{\gamma}) \frac{\partial v_{i}}{\partial x_{j}}\right]=-\frac{\partial p}{\partial x_{i}}+\frac{\partial}{\partial x_{j}}\left[\eta(\dot{\gamma}) \frac{\partial v_{j}}{\partial x_{i}}\right]
\end{gathered}
$$

As usually $v_{i}$ denotes the velocity vector, $x_{i}$ the Cartesian coordinate, $\rho$ the density of a fluid and $p$ the pressure. Since the fluid flow is studied as a two-dimensional no flow occurs in the $z$ direction, and no flow variable depends on the $z$ co-ordinate, i.e. $i, j=x, y$ and $\partial \phi / \partial z=0$ for all flow variables $\phi$.

In a momentum conservation equation (Eq. 2) the constitutive (rheological) model is needed for the viscous function $\eta(\dot{\gamma})$. In a case of generalized Newtonian fluids, the form of general relationship between the shear stress and shear rate is:

$$
\tau_{i j}=\eta(|\dot{\gamma}|) \dot{\gamma}_{i j}
$$


where $|\dot{\gamma}|=\sqrt{(1 / 2) \sum_{i} \sum_{j} \dot{\gamma}_{i j} \dot{\gamma}_{j i}}$ stands for the II. invariant of the symmetrical rate of deformation tensor with Cartesian components $\dot{\gamma}_{i j}=\left(\partial v_{i} / \partial x_{j}\right)+\left(\partial v_{j} / \partial x_{i}\right)[6]$.

\subsection{Rheology of a working fluid}

As an example of the shear-thinning fluid, a human blood was taken under consideration. One of the generally accepted properties of blood is its shear-thinning behaviour. Several formulas have been used and calibrated for the use in the blood flow simulations.

Experimental results for the shear rate and the shear stress obtained with the shearrheometer in a Couette geometry [7] were used to accommodate widely used and highly successful five-parameter Carreau-Yasuda model, given by:

$$
\eta(\dot{\gamma})=\eta_{\infty}+\frac{\eta_{0}-\eta_{\infty}}{\left[1+(\lambda \dot{\gamma})^{b}\right]^{a}}
$$

with the following value of constants [8]: $\eta_{0}=1.612 \cdot 10^{-1} \mathrm{Pas}, \quad \eta_{\infty}=3.527 \cdot 10^{-3} \mathrm{Pas}$, $\lambda=8.2 s, a=1.23$ and $b=0.64$.

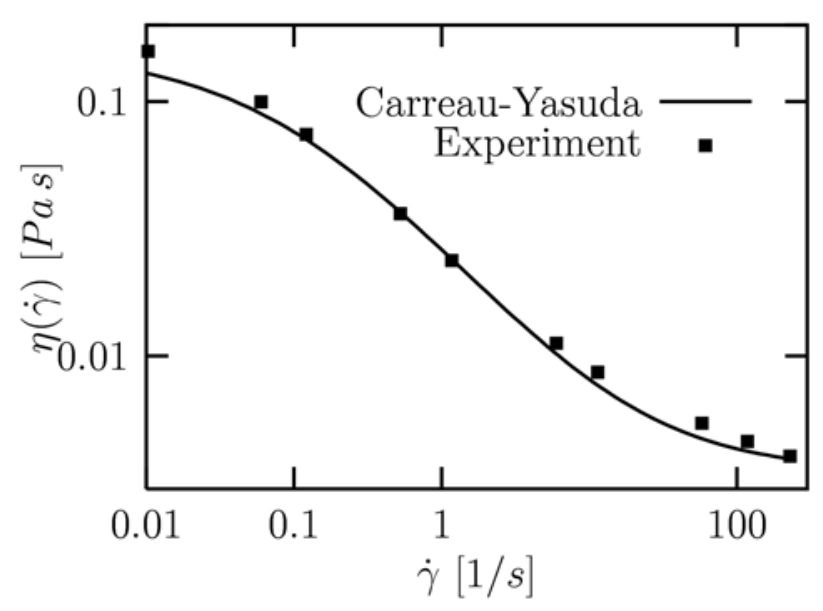

Figure 1: Human blood rheology; Carreau-Yasuda model versus experimental data.

In order to compare the influence of a shear-thinning viscous behaviour on the symmetry breaking phenomena, the blood flow in a locally constricted channel was also examined by numerical means as the Newtonian fluid, taking the viscosity equal to a high-shear limiting viscosity of a Carreau-Yasuda model $\left(\eta=\eta_{\infty}=3.527 \cdot 10^{-3} \mathrm{Pas}\right)$. It is a value very similar to the values that were commonly used in previous studies of blood flows; e.g. $\eta=3.45 \cdot 10^{-3}$ Pas [9] and $\eta=3.50 \cdot 10^{-3}$ Pas [10].

\section{GEOMETRY AND BOUNDARY CONDITIONS}

Since no expression for the generalized Reynolds number for Carreau-Yasuda fluid flow in a channel exists in a literature, it was determined throughout the analysis of a Newtonian and Carreua-Yasuda fluid flow between parallel plates, Fig. 2. 
As in the work of [4], the boundary conditions for a Newtonian fluid channel flow were set according to the Newtonian Reynolds number $\operatorname{Re}=(\rho \bar{v} H) / \eta$ and were also imposed for the Carreau-Yasuda fluid.

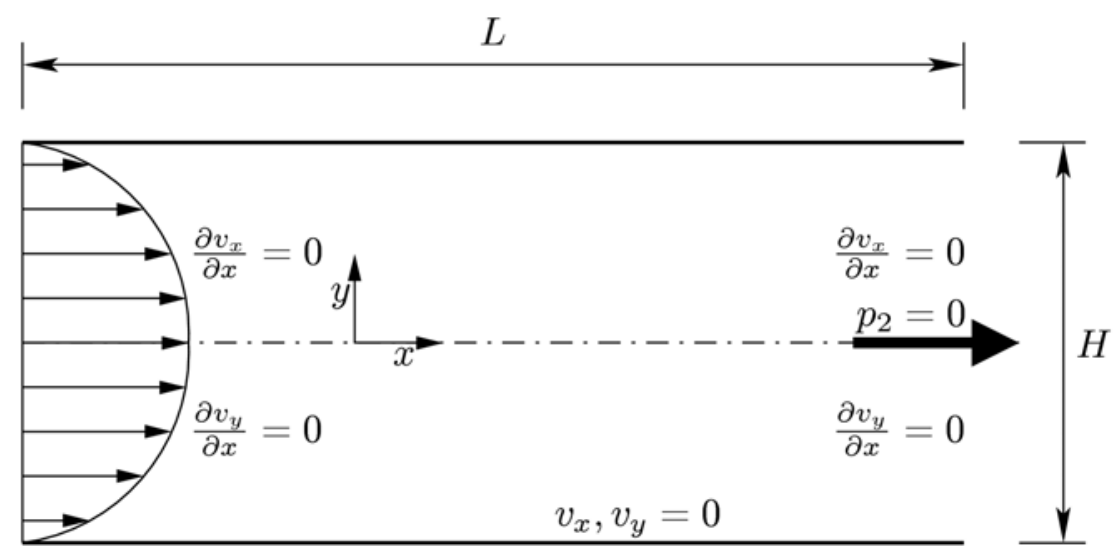

Figure 2: Flow between parallel plates; $H=1, L=5 \times H$.

The geometry of a locally constricted channel consists of an upstream and downstream section as depicted in Fig. 3. The inlet to the flow domain is located some distance $\left(L_{u}\right)$ upstream of the narrowest part (this is taken as the coordinate origin, $x=0$ ), while the outlet is located far downstream $\left(L_{d}\right)$ from that part. The generalized Reynolds number was defined as for the channel flow, i.e. the inlet channel height $(H)$ was taken as a characteristic length and the average velocity in the upstream channel $(\bar{v}=1)$ as the characteristics velocity scale.

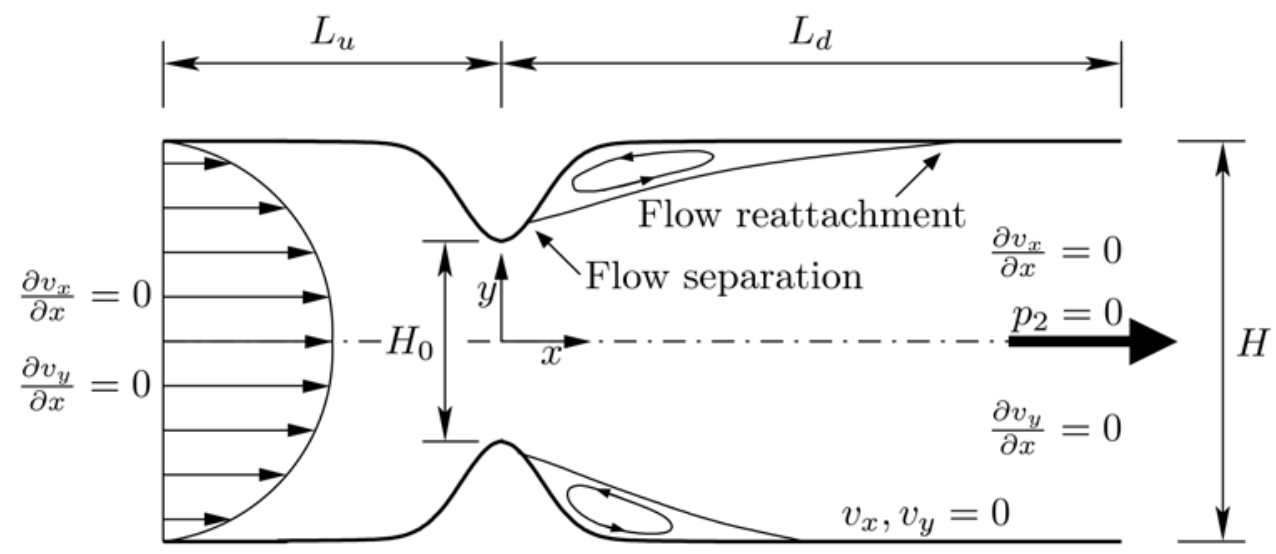

Figure 3: Locally constricted channel flow geometry with expected flow patterns; $H=1, L_{u}=5 \times H, L_{d}=35 \times H$.

Geometrical model of a constricted channel, as shown in Fig. 3, includes a local constriction defined by the Gaussian equation:

$$
\frac{H(x)}{H_{0}}=1-\frac{1}{2} \exp \left(-\frac{4 x^{2}}{H_{0}^{2}}\right)
$$

where $H(x)$ is the local height of a constricted channel and $H_{0}=H / 2$.

To completely define the flow problem, boundary conditions for the velocity and pressure fields must be specified. At the inflow boundary, fully developed flow conditions were 
considered by imposing the Neumann boundary conditions on a velocity while the pressure is derived by linear extrapolation from the inner nodes. For both geometries, the outlet boundary conditions were used to ensure the fully developed flow conditions with a combination of Neumann (zero normal velocity gradients) and Dirichlet boundary conditions (fixed value for the pressure). The latter (obtaining the pressure field inside the domain by specifying the reference value) is a common practice since the pressure field obtained by solving the pressure-correction equation does not give absolute pressures [11].

\section{NUMERICAL METHOD}

The governing equations were solved using CFX-4.4 computer code which employs the standard finite volume method with all variables defined at the centre of the control volumes populating the physical domain under consideration. Each equation is integrated over each control volume to obtain a discrete equation which connects the variable at the centre of the volume with its neighbours.

For a spatial discretization the Quadratic upwind differencing scheme [12] was used. It is an upwind scheme (using two upstream points and one downstream point) which is a thirdorder accurate for the advection terms, while all other (diffusion) terms remain second-order.

The mass and momentum equations in two dimensions provide three unknowns: $v_{x}, v_{y}$, and $p$. In the solution process, an equation describing the update of the pressure is required but is not explicitly available via the mass or momentum balances. Updated velocity and pressure fields satisfying exactly mass balance and approximately satisfying the discrete momentum equations were obtained by the SIMPLEC algorithm [13], which resolves the coupling between the velocity and pressure.

\section{RESULTS AND DISCUSSION - CHANNEL FLOW}

A brief numerical analysis of a Newtonian and shear-thinning fluid flow in a straight channel was performed in order to define the analytical expression for the generalized Reynolds number for the Carreua-Yasuda fluid $\left(R e_{C Y}\right)$.

\subsection{Nodalization analysis}

The influence of a computational mesh refinement on numerical results was studied throughout the examination of spatial (grid) convergence. For this, the Newtonian and Carreau-Yasuda fluid flow at Newtonian Reynolds number $R e=250$ were studied using three computational meshes with uniformly distributed elements, Table I.

Table I: Computational mesh characteristics, nodalization analysis and estimation of numerical accuracy for Newtonian $(N)$ and Carreau-Yasuda $(C Y)$ fluid channel flow.

\begin{tabular}{|c|c|c|c|}
\hline Mesh & Number of elements & $\Delta p_{N}[\mathrm{~Pa}]$ & $\Delta p_{C Y}[\mathrm{~Pa}]$ \\
\hline Mesh I & $50 \times 10$ & 0.210634 & 0.733502 \\
\hline Mesh II & $100 \times 20$ & 0.211406 & 0.715618 \\
\hline Mesh III & $200 \times 40$ & 0.211563 & 0.710664 \\
\hline$\Phi$ & $/$ & 0.211573 & 0.710334 \\
\hline$E_{r}[\%]$ & $/$ & 0.0047 & 0.0465 \\
\hline
\end{tabular}


The numerical accuracy was assessed taking the advantage the Richardson's extrapolation technique. It is a method for obtaining-higher order estimate of the flow value (value at infinite grid) from a series of lower-order discrete values. The extrapolated values (to a zero mesh size) were determined as:

$$
\Phi=\frac{\left(N_{I I I} / N_{I I}\right)^{o s} \Phi_{I I I}-\Phi_{I I}}{\left(N_{I I I} / N_{I I}\right)^{o s}-1}
$$

and the relative error as:

$$
E_{r}=\left|\frac{\Phi_{I I I}-\Phi}{\Phi}\right| \times 100
$$

Here, $\Phi_{I I}$ and $\Phi_{I I I}$ are values of particular flow variable under investigation obtained with Mesh II and Mesh III, $N_{I I}$ and $N_{I I I}$ the numbers of elements for particular mesh, and os is the order of scheme (os $=2$ for diffusion terms).

The variation of an overall all pressure drop for both, Newtonian and shear-thinning Carreau-Yasuda fluid channel flow with grid refinement (increasing number of elements) is given in tabulated form in Table I. The '\% error' given in Table I is a quantification of the relative difference between the prediction of particular pressure drop on the computational Mesh III and the extrapolated result. In effect, it is a measure of an uncertainty of present numerical results. As can be seen, the differences with grid refinement (i.e. Mesh II and Mesh III) are exceeding small and the agreement between Mesh III and extrapolated value is extremely good with the numerical error well below $0.05 \%$.

\subsection{Generalized Reynolds number for Carreau-Yasuda fluid}

Normalized pressure drop for the Newtonian fluid channel flow can be expressed as [4, 14]:

$$
\frac{H \Delta p_{N}}{L \rho \bar{v}^{2}}=\frac{12}{\operatorname{Re}_{N}}
$$

and one can write down the following for $R e_{C Y}$ :

$$
\operatorname{Re}_{C Y}=12 \frac{L}{H} \frac{\rho \bar{v}^{2}}{\Delta p_{C Y}}
$$

Since the same geometry of a channel, physical properties and inlet average velocity were used for the Newtonian and Carreau-Yasuda fluid, the following expression is derived:

$$
\frac{\operatorname{Re}_{C Y}}{\operatorname{Re}_{N}}=\frac{\Delta p_{N}}{\Delta p_{C Y}} \Rightarrow \operatorname{Re}_{C Y}=0.297698 \cdot \operatorname{Re}_{N}
$$

and it was found to be valid for the entire range of $R e_{N}$ as shown by the Fig. 4 .

Present estimate of a generalized Reynolds number for the Carreau-Yasuda fluid, Eq. 9, is a reflection of boundary conditions $(\bar{v}=1)$ and geometry used in the channel flow analysis. Therefore, if any of both parameters changes ( $\bar{v}$ or $H$ ), so will the ratio $R e_{C Y} / \operatorname{Re}_{N}$. 


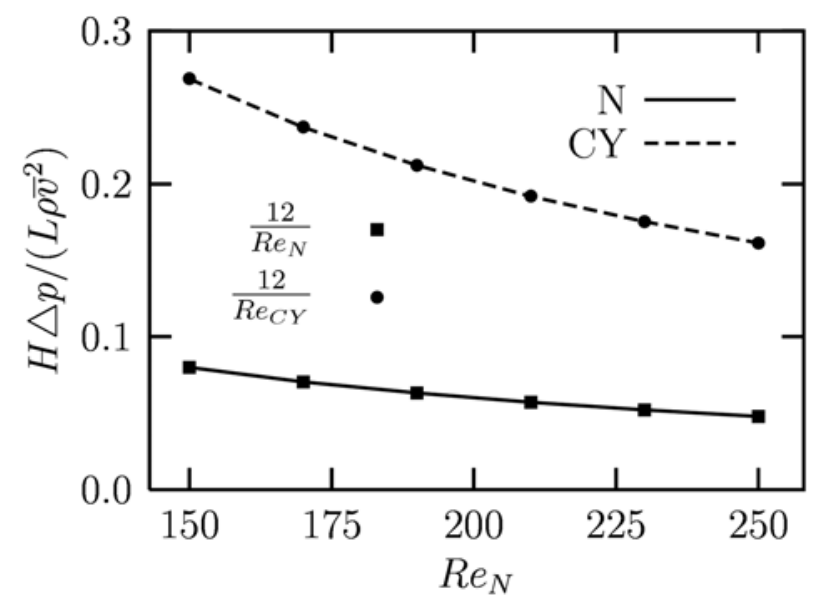

Figure 4: Normalized pressure variation for Newtonian (N) and Carreau-Yasuda (CY) fluid.

\section{RESULTS AND DISCUSSION - CONSTRICTED CHANNEL FLOW}

\subsection{Nodalization analysis}

The aim of most numerical analysis is to achieve certain accuracy within the smallest amount of a computational work. The difficulty to accomplish this lies in the unknown behaviour of the flow under numerical investigation. The influence of computational grid refinement on numerical results was studied throughout the examination of spatial (grid) convergence. For this the Newtonian fluid flow at generalized Reynolds number $R e=250$ was studied using three different computational meshes (Table II) with the grid refinement ratio 4.

Table II: Computational mesh characteristics, nodalization analysis and estimation of numerical accuracy for Newtonian fluid flow in a locally constricted channel.

\begin{tabular}{|c|c|c|c|}
\hline Mesh & Number of elements & $X_{R 1}^{* *} / H$ & $X_{R 2}^{* *} / H$ \\
\hline Mesh I & $400 \times 10$ & 1.2396 & 1.2405 \\
\hline Mesh II & $800 \times 20$ & 1.5433 & 3.3907 \\
\hline Mesh III & $1600 \times 40$ & 1.6878 & 3.4711 \\
\hline$\Phi$ & $/$ & 1.6974 & 3.4765 \\
\hline$E_{r}[\%]$ & $/$ & 0.5675 & 0.1542 \\
\hline
\end{tabular}

The numerical accuracy of a constricted channel flow was assessed as for the straight channel flow using the Eq. 5 (for extrapolated values) and Eq. 6 (for numerical error). The variation of the normalized reattachment length of both, larger and smaller vortex $\left(X_{R 1}^{* *} / H\right.$, $\left.X_{R 2}^{* *} / H\right)$ with grid refinement is given in Table II. As can be seen, the differences with increasing number of elements (i.e. Mesh II and Mesh III) are exceeding small and the agreement between Mesh III and extrapolated values is excellent (numerical error much lower than $0.6 \%)$.

Based on the results presented in Table II we conclude that further grid refinement would not result in a significantly improved numerical accuracy. In addition to that, the computational Mesh III was found to yield numerical results that are independent of the mesh density (number of elements populating the physical domain), as presented by Fig. 5 for the normalized velocity distribution at two axial positions in a reversal flow regime. 

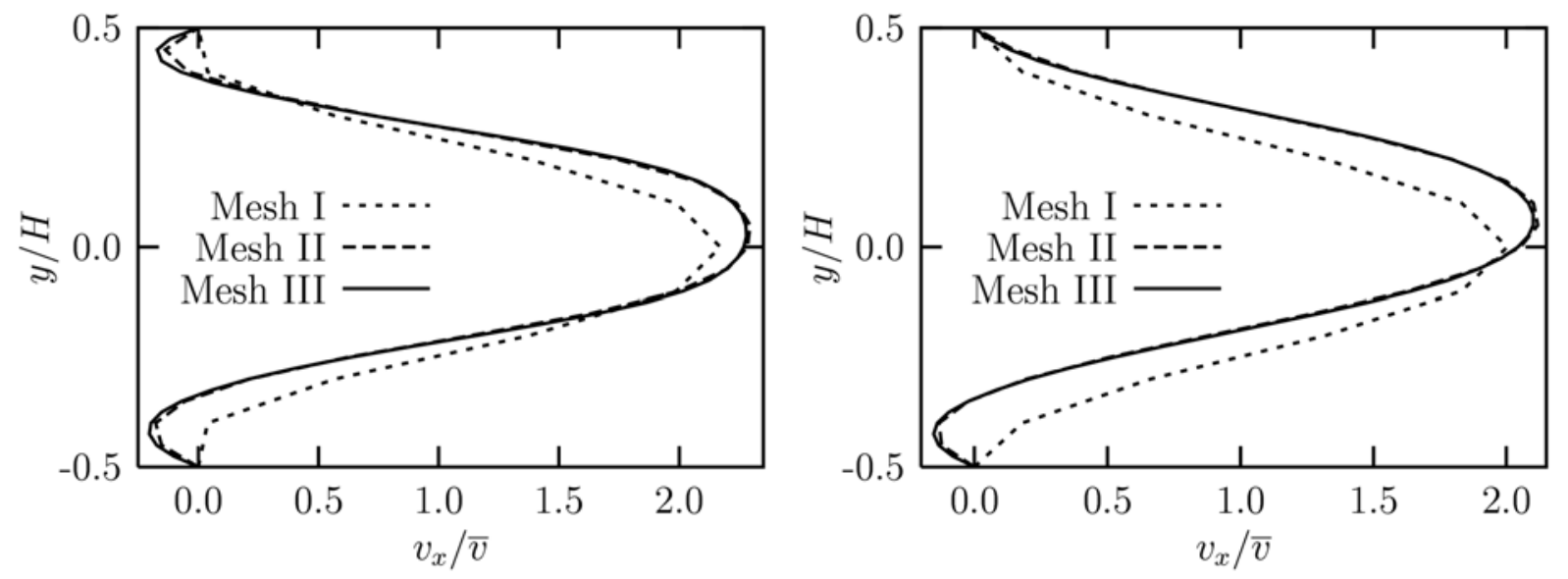

Figure 5: Normalized axial velocity distribution for Newtonian fluid; $x=1 \times H$ (left) and $x=2 \times H$ (right).

Results for the computational Mesh III can be misleading since the main flow is pushed toward the upper wall while for Mesh I and Mesh II the main flow is pushed toward the lower wall. But this observation is in agreement with other studied where the random appearance of the larger vortex along lower or upper wall was reported for sudden expansion flows of Newtonian $[4,15]$ as well as non-Newtonian fluids [16].

\subsection{Symmetry breaking phenomena}

Bifurcation diagrams illustrate the transition from symmetric to asymmetric fluid flow in terms of the difference $\left(D_{X}\right)$ between the size of a smaller and larger vortex $\left(X_{R 1}=X_{R 1}^{* *}-X_{R 1}^{*}\right.$ and $\left.X_{R 2}=X_{R 2}^{* *}-X_{R 2}^{*}\right)$. Every fluid model exhibits a different bifurcation diagram in which $D_{X}$ depends on the parameters of particular model. In a present study, the bifurcation phenomenon is studied from the view of vortex characteristics and bifurcation diagram.

Vortex characteristics and bifurcation diagram for the reversal flow are presented in Fig. 6. Initially, at lower values of generalized Reynolds number, the size of both vortices is equal for particular fluid, indicating the symmetric flow conditions. At a critical value of generalized Reynolds number $\left(R e_{N, c r}=220, R e_{C Y, c r}=216\right)$ there is a branching that indicates asymmetric flow. Immediately after the occurrence of flow asymmetry, there is a rapid change in length of both vortices and as the generalized $R e$ number further increases the smaller vortex $\left(X_{R 1}\right)$ remains relatively constant while the larger vortex on the opposite channel wall $\left(X_{R 2}\right)$ starts to increase more rapidly; the recirculation region characterized by $X_{R 2}$ continues to grow on the expense of the other recirculation zone $X_{R 1}$ and this is observable only for the shear-thinning (Carreau-Yasuda) fluid $R e_{C Y} \geq 235$.

Direct comparison of vortex characteristics for both fluids is given in Fig. 7, which is obtained by modifying Fig. 6 in such a way, that the critical value of generalized Reynolds number is taken as a reference point.

In a symmetric flow regime ( $R e \leq R e_{c r}$ ), the variation of vortices length versus generalized Reynolds number is more inclined for the shear thinning (Carreau-Yasuda) fluid in comparison to the Newtonian fluid. As the Reynolds number further increases (asymmetric flow regime $R e \geq R e_{c r}$ ) the larger vortex grows more rapidly for Carreau-Yasuda fluid as presented by Fig. 7. Similar, i.e. more rapid change, is observed for the length of a smaller vortex. Immediately after the point of bifurcation, the decrease in $X_{R 1}$ is more pronounced for the shear-thinning fluid, and as the Reynolds number further increases both fluids (Newtonian and Carreau-Yasuda) start to exhibit similar variation of smaller vortex, Fig. 7. 

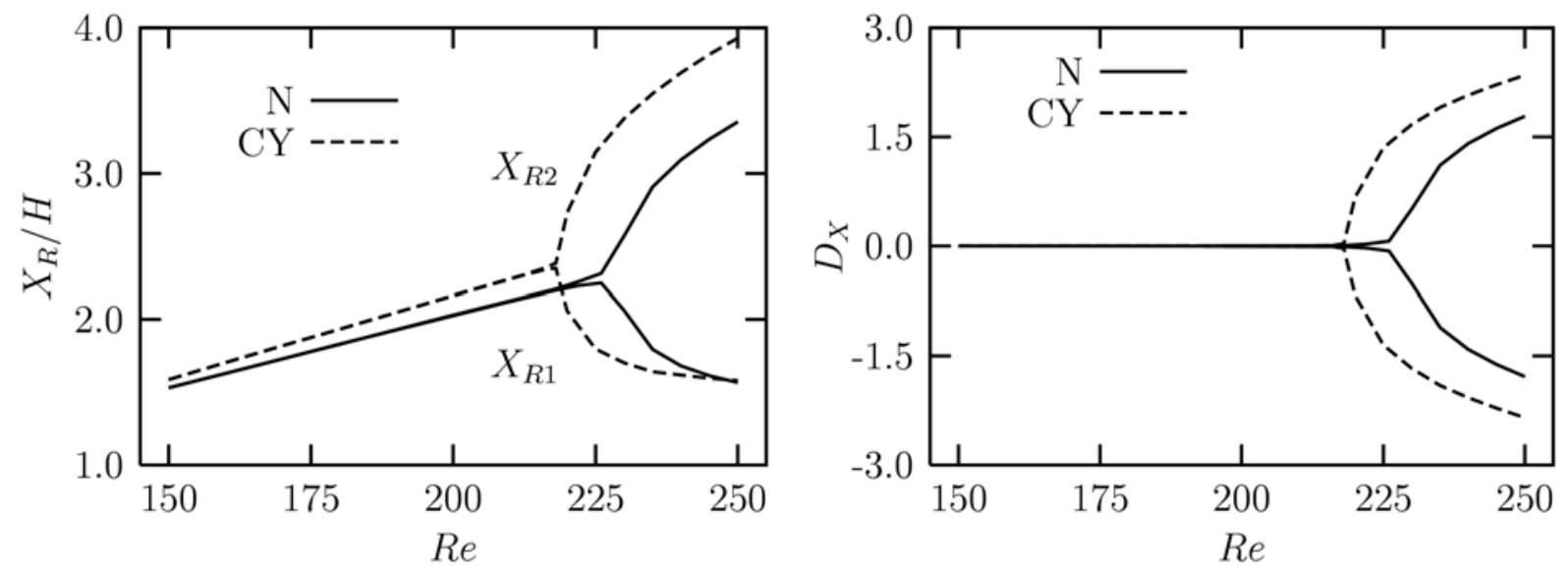

Figure 6: Vortex flow characteristics for Newtonian (N) and Carreau-Yasuda (CY) fluid; size of vortices (left) and bifurcation diagram (right).

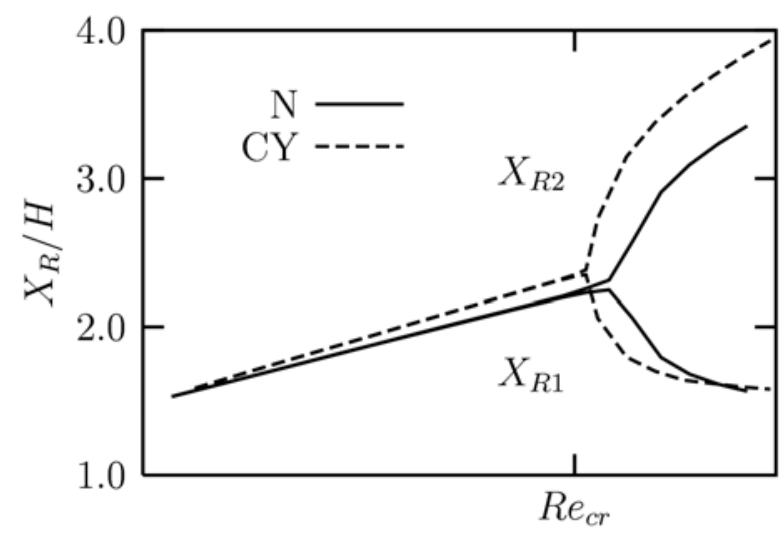

Re

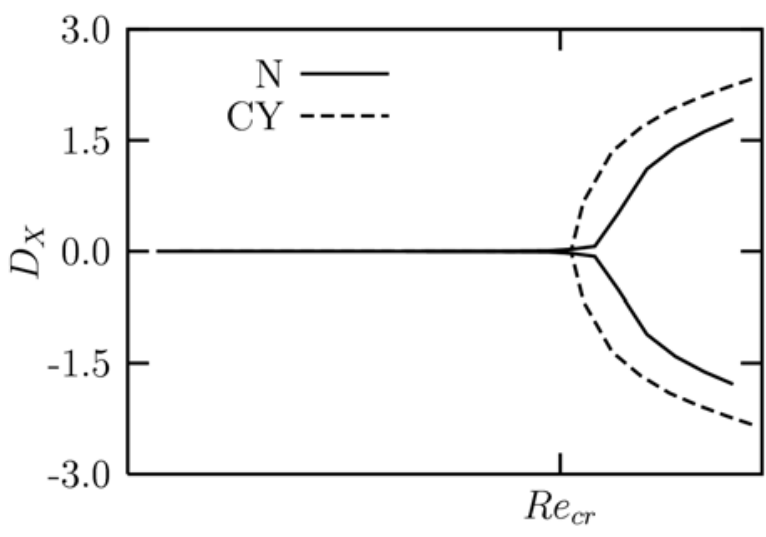

Re

Figure 7: Vortex flow characteristics in the modified diagram for Newtonian (N) and CarreauYasuda (CY) fluid; size of vortices (left) and bifurcation diagram (right).

Shear-thinning viscous behaviour has a significant influence not only on the occurrence flow asymmetry (bifurcation point) but also on the size of vortices at the point of bifurcation. In comparison to the Newtonian fluid, the critical value of generalized Reynolds number is decreased, but on the other hand the normalized length of vortices at the point of transition from flow symmetry to asymmetry is found to increase for the shear-thinning viscous behaviour, Fig. 6 and Table III.

Table III: Characteristical values at point of bifurcation.

\begin{tabular}{|c|c|c|c|}
\hline & $\mathrm{Re}_{c r}$ & $X_{R 1} / H$ & $X_{R 2} / H$ \\
\hline Newtonian fluid & 220 & 2.2128 & 2.2316 \\
\hline Carreau-Yasuda fluid & 216 & 2.3376 & 2.3488 \\
\hline
\end{tabular}

In contrast to sudden expansion flows, where the flow separates immediately in the expansion plane $(x=0)$, present geometry results in a slight shift of flow separation away from the narrowest part of a restriction $(x>0)$, left hand side of Fig. 8 and 9. For both, smaller and larger vortex the separation point is approaching the narrowest part of a restriction as the 
generalized Reynolds number increases and is closer to the narrowest of a restriction for the shear-thinning fluid.
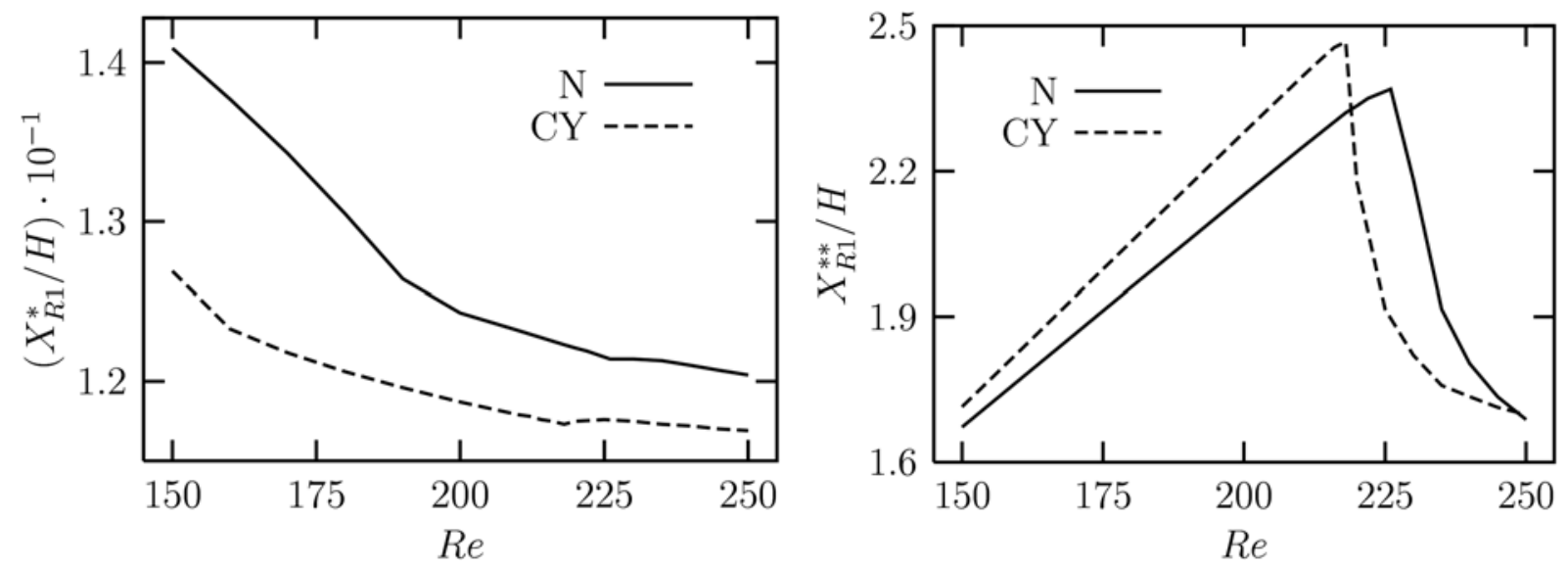

Figure 8: Smaller vortex characteristics for Newtonian (N) and Carreau-Yasuda (CY) fluid; separation (left) and reattachment point (right).
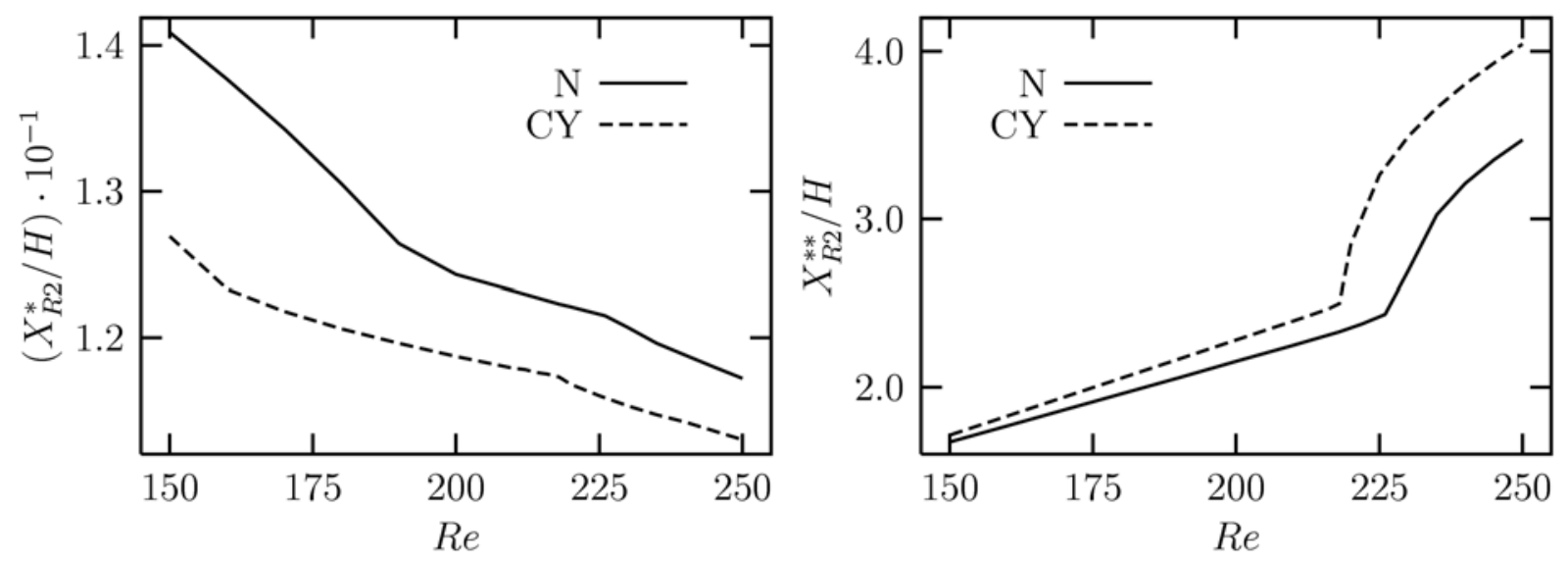

Figure 9: Larger vortex characteristics for Newtonian (N) and Carreau-Yasuda (CY) fluid; separation (left) and reattachment point (right).

On the other hand, the shear-thinning viscous behaviour increases the reattachment point for smaller as well as larger vortex in the symmetric flow regime $\left(R e \leq R e_{c r}\right)$, right hand side of Fig. 8 and 9. As the Reynolds number further increases there is sudden change in variation of the reattachment position versus the Reynolds number; variation is no longer linear and smaller vortex is reattached later for the Newtonian fluid, while the larger vortex is reattached later for the shear-thinning fluid.

\section{CONCLUSIONS}

In a present study, the steady and laminar flow of a generalized Newtonian fluid in the twodimensional channel with local constriction was studied numerically using mathematical modelling and a finite volume based CFD code. The shear-thinning viscous behaviour of a working fluid (human blood) has been considered with the well known and widely used fiveparameter Carreau-Yasuda model and the numerical results are compared with the Newtonian fluid. 
Present research is prompted by the fact that most of the research activities (numerical as well as experimental) where the fluid flow experiences the transition from symmetry to asymmetry involve channels with sudden and symmetrical expansions with various expansion ratios and that no computational studies regarding the generalized Newtonian fluid flow through a channel with local constriction has been reported so far.

First, the numerical analysis of Newtonian and Carreau-Yasuda fluid flow in a straight channel was performed and resulted in an estimate of generalized Reynolds number for the Carreau-Yasuda fluid.

A systematic nodalization analysis, using three computational meshes was performed to study the influence of grid refinement on numerical results for the channel and expansion flow. For the channel flow, Richardson's extrapolation technique was used to quantify the numerical accuracy for the pressure drop while the normalized reattachment length of both vortices was considered for the locally constricted channel flow. The estimated numerical accuracy is well below $0.05 \%$ for the channel flow and $0.6 \%$ for the flow in a constricted channel.

In a range of generalized Reynolds number $150 \leq R e \leq 250$ for particular generalized Newtonian fluid the flow in a locally constricted channel was thoroughly examined with a focus on the transition of symmetry to asymmetry and the effect of a shear-thinning viscous behaviour on the this phenomena. From the numerical results for Newtonian and shearthinning Carreau-Yasuda fluid, the following conclusions can be emphasized:

- Transition from flow symmetry to its asymmtery is greatly affected by the shear-thinning fluid behaviour. Not only that the critical value of generalized Reynolds number is decreased in comparison to the critical value for Newtonian fluid, the length of vortices for the shear-thinning fluid varies differently with the generalized Reynolds number.

- In the symmetric flow regime the variation of vortices length $\left(X_{R 1}\right.$ and $\left.X_{R 2}\right)$ versus generalized Reynolds number is more inclined and the vortices length at the bifurcation point is greater for the shear-thinning Carreau-Yasuda fluid.

- After the transition from flow symmetry to asymmetry there is a rapid change in length of both vortices and as the generalized Re number further increases the smaller vortex $\left(X_{R 1}\right)$ remains relatively constant while the larger vortex on the opposite channel wall $\left(X_{R 2}\right)$ starts to grow more rapidly on the expense of a smaller vortex.

- In contrast to the sudden expansion flows where the point of separation is fixed, the flow separation is shifted away from the narrowest part of restriction (this shift is more pronounced for the Newtonian fluid) and this shift decreases as the generalized Reynolds number increases.

- In a symmetric flow regime the shear-thinning fluid flow reattaches later in comparison to the Newtonian fluid, and as the Reynolds number further increases (asymmetric flow regime) smaller vortex is reattached later for the Newtonian fluid, while the larger vortex is reattached later for the shear-thinning fluid

\section{REFERENCES}

[1] Durst, F.; Melling, A.; Whitelaw, J. H. (1974). Low Reynolds number flow over a plane symmetrical sudden expansion, Journal of Fluid Mechanics, Vol. 64, 111-128

[2] Fearn, R. M.; Mullin, T.; Cliffe, K. A. (1990). Nonlinear flow phenomena in a symmetric sudden expansion, Journal of Fluid Mechanics, Vol. 211, 595-608

[3] Bell, B. C.; Surana, K. S. (1994). p-version least squares finite element formulation for twodimensional, incompressible, non-Newtonian isothermal and non-isothermal fluid flow, International Journal for Numerical Methods in Fluids, Vol. 18, 127-162 
[4] Ternik, P.; Marn, J.; Žunič, Z. (2006). Non-Newtonian fluid flow through a planar symmetric expansion: Shear-thickening fluids, Journal of Non-Newtonian Fluid Mechanics, Vol. 135, 136148

[5] Mishra, S.; Jayaraman, K. (2002). Asymmetric flows in planar symmetric channels with large expansion ratio, International Journal for Numerical Methods in Fluids, Vol. 38, 945-962

[6] Bird, R. B.; Stewart, W. E.; Lightfoot, E. N. (1960). Transport Phenomena, John Wiley \& Sons, Inc., USA

[7] Arora, D. (2006). Computational Hemodynamics: Hemolysis and Viscoelasticity (PhD Thesis), USA.

[8] Ternik, P.; Marn, J. (2009). Numerical study of blood flow in stenotic artery, Applied Rheology, Vol. 19, accepted manuscript (article in production)

[9] Johnston, B. M.; Johnston, P. R.; Corney, S.; Kilpatrick, D. (2004). Non-Newtonian blood flow in human right coronary arteries: Steady state simulations, Journal of Biomechanics, Vol. 37, 709-720

[10] Shibeshi, S. S.; Collins, W. E. (2005). The rheology of blood flow in a branched arterial system, Applied Rheology, Vol. 15, 398-405

[11] Patankar, S. V. (1980). Numerical Heat Transfer and Fluid Flow, Hemisphere Publishing Corporation, Taylor \& Francis Group, New York

[12] Leonard, B. P. (1979). A Stable and Accurate Convective Modeling Procedure Based on Quadratic Upstream Interpolation, Computer Methods in Applied Mechanics and Engineering, Vol. 19, 59-98

[13] Van Doormaal, J. P.; Raithby, G. D. (1984). Enhancements of the SIMPLE algorithm for predicting incompressible fluid flows, Numerical Heat Transfer, Vol. 7, 147-163

[14] Oliveira, P. J. (2003). Asymmetric flows of viscoelastic fluids in symmetric planar expansion geometries, Journal of Non-Newtonian Fluid Mechanics, Vol. 114, 33-63

[15] Foumeny, E. A.; Ingham, D. B.; Walker, A. J. (1996). Bifurcations of incompressible flow through plane symmetric channel expansions, Computers \& Fluids, Vol. 25, 335-351

[16] Neofytou, P.; Drikakis, D. (2003). Non-Newtonian flow instability in a channel with sudden a sudden expansion, Journal of Non-Newtonian Fluid Mechanics, Vol. 111, 127-150 